7090 errors, four BESK errors (2957, 2969, 3049 and 3109), and an incorrect SWAC result for 1889. The SWAC (October 1962) confirmed the 7090 residue.

UCLA Computing Facility

University of California, Los Angeles

1. Alexander Hurwitz, "New Mersenne primes," Math. Comp., v. 16, 1962, p. 249-251.

2. A. HuRwitz \& J. L. Selfridge, "Fermat numbers and perfect numbers," Notices Amer. Math. Soc., v. 8, 1961, p. 601, abstract 587-104.

3. G. A. PAXson, "The compositeness of the thirteenth Fermat number," Math. Comp., v. 15,1961, p. 420 .

4. HaNs Riesel, "Mersenne numbers," MTAC, v. 12, 1958, p. 207-213.

5. Raphael M. Robinson, "Mersenne and Fermat numbers," Proc. Amer. Math. Soc., v. 5,1954 , p. $842-846$.

\title{
Lucas' Test for Mersenne Numbers, $6000<p<7000$
}

\section{By Sidney Kravitz and Murray Berg}

Alexander Hurwitz [1] reported that he had applied Lucas' test to investigate the primality of the Mersenne Numbers $M_{p}=2^{p}-1, p$ a prime, $3300<p<5000$, and discovered that $M_{4253}$ and $M_{4423}$ are prime numbers. Hurwitz [2] further states $\dagger$ that he tested all prime exponents between 5000 and 6000, where the corresponding $M_{p}$ was not known to have a factor, without discovering any new Mersenne Primes.

\begin{tabular}{cccccc}
\multicolumn{7}{c}{ TABLE } \\
\hline$p$ & $R$ & $p$ & $R$ & $p$ & $R$ \\
\hline 6007 & 07707 & 6247 & 00472 & 6659 & $75241^{*}$ \\
6037 & 21420 & 6257 & 36710 & 6661 & 27165 \\
6043 & 21605 & 6269 & 57356 & 6679 & 13275 \\
6047 & 37000 & 6299 & $71037^{*}$ & 6701 & 07636 \\
6053 & 53471 & 6329 & $25136^{*}$ & 6709 & 05700 \\
6073 & 41646 & 6337 & $21676^{*}$ & 6733 & 35544 \\
6079 & 15712 & 6359 & 51351 & 6763 & 01753 \\
6089 & 32615 & 6361 & $10027^{*}$ & 6779 & $74306^{*}$ \\
6091 & 02043 & 6451 & 23476 & 6791 & 41143 \\
6133 & 42630 & 6469 & 51252 & 6823 & $14573^{*}$ \\
6151 & 63451 & 6547 & 06546 & 6833 & 26431 \\
6211 & 71252 & 6571 & 67142 & 6857 & 63102 \\
6217 & 07377 & 6577 & $45051^{*}$ & 6907 & $46461^{*}$ \\
6221 & 24166 & 6581 & $7420^{*}$ & 6911 & 63345 \\
6229 & 06517 & 6599 & 77554 & 6971 & 65345 \\
& & & & 6991 & 50365 \\
\hline
\end{tabular}

The authors have tested the Mersenne Numbers $6000<p<7000$ without finding any new primes. A list of the five least significant octal digits of the $S_{p-1}$ th remainder from the Lucas test $\left(S_{1}=4\right)$ is given in the Table. Where a prime is missing from the list it indicates that a factor of the corresponding Mersenne Number was found by Riesel [3,4] or that an unpublished $\dagger$ factor was found by

Received February 6, 1963. Revised April 26, 1963.

$\dagger$ See pages 146, 87, and 93 of this issue of Mathematics of Computation. 
John Brillhart. At the time of cornpletion of these results we learned of similar work $\dagger$ by Donald B. Gillies on Illiac II. We compared our residues with his and found ten discrepancies. A check revealed that one of our three supposedly identical program decks contained an error. The questionable residues were recalculated and found to agree with Dr. Gillies' values. These residues are marked by an asterisk $\left({ }^{*}\right)$.

The authors have verified that Riesel's $M_{3217}$ and Hurwitz's $M_{4253}$ and $M_{4423}$ are prime. Hurwitz's octal remainder [1] of 72013 for the prime exponent 3301 was also verified. The running time for $p$ near 6500 was three hours, using an IBM 7090.

Picatinny Arsenal

Dover, New Jersey

Standard Oil Company of California

San Francisco, California

1. A. Hurwitz, "New Mersenne primes", Math. Comp., v. 16, 1962, p. 249-51.

2. A. Hurwitz, Private communication to the authors dated March 12, 1962.

3. H. Riesel, "Mersenne numbers", $M T A C$, v. 12, 1958, p. 207.

4. H. Riesel, "All factors $q<10^{8}$ in all Mersenne numbers, $2^{p}-1, p$ a prime $<10^{4}$ ", Math. Comp., v. 16, 1962, p. 478-82. Errata, Math. Comp., v. 17, 1963, p. 486.

5. S. Kravitz \& M. Berg, "Recent research in Mersenne numbers" Recreational Mathematics Magazine, October, 1962. p. 40.

\section{Note on the Congruence $a^{p-1} \equiv 1\left(\bmod p^{2}\right)$.}

\section{By Hans Riesel}

During 1961 the author with the aid of the electronic computer BESK gathered some data concerning the residues

$$
a^{p-1}\left(\bmod p^{2}\right)
$$

for odd primes $p$, from which the following tables have been compiled. In the case of $p<1000$, the residues of $a^{p-1}\left(\bmod p^{2}\right)$ are available for comparison. For larger $p$ the program has printed out only those residues $\equiv 0\left(\bmod p^{2}\right)$. The running time on BESK was put at the author's disposal by courtesy of the Swedish Board for Computing Machinery.

Mabarsstigen 2

Stockholm-Vallingby, Sweden

All odd primes $p<500000$ with $a^{p-1} \equiv 1\left(\bmod p^{2}\right)$ for $a \leqq 10$.

\begin{tabular}{clrl}
\hline$a$ & \multicolumn{1}{c}{$p$} & $a$ & \multicolumn{1}{c}{$p$} \\
\hline 2 & 1093,3511 & 6 & 66161 \\
3 & 11 & 7 & 5,491531 \\
4 & 1093,3511 & 8 & $3,1093,3511$ \\
5 & 20771,40487 & 9 & 11 \\
& & 10 & 3,487 \\
\hline
\end{tabular}

Received May 27, 1963. 